\title{
Formulação de fluxo em arcos para problemas de agrupamento capacitado
}

\author{
Vítor G. Chagas ${ }^{1}$, Manuel Iori ${ }^{2}$ \\ ${ }^{1}$ Instituto de Computação - Universidade Estadual de Campinas (UNICAMP) \\ Cidade Universitária Zeferino Vaz - Barão Geraldo, Campinas, SP \\ ${ }^{2}$ DISMI - University of Modena and Reggio Emilia (UNIMORE) \\ Via Amendola 2, 42122 Reggio Emilia, Itália
}

\begin{abstract}
In this work, we present a formulation based on arc-flow models, originally designed for bin packing problems, and now applied to clustering problems. We consider the Correlation Clustering and Graph Partitioning problems with node weights and a cost associated to each cluster. We discuss the advantages of this formulation in comparison with other strategies presented in the literature.
\end{abstract}

Resumo. Neste trabalho, apresentamos uma formulação baseada em modelos de fluxo em arcos, originalmente projetados para problemas de empacotamento, e agora aplicada a problemas de agrupamento. Consideramos os problemas de Correlation Clustering e Graph Partitioning com pesos nos vértices e custo associado a cada cluster utilizado. Discutimos as vantagens de tal formulação comparada a outras estratégias presentes na literatura.

\section{Introdução}

O problema de agrupar um conjunto de dados que possuem informações similares é um problema com bastante interesse teórico e prático, possuindo aplicações em várias áreas, como mineração de dados, biologia computacional, compressão de dados, aprendizado de máquina, reconhecimento de padrões e visão computacional.

Um tipo de problema de agrupamento (clustering) é o Correlation Clustering (CC), onde é dado um grafo $G=(V, E)$ tal que cada aresta $e \in E$ possui um sinal $s(e) \in\{+,-\}$. A versão de minimização de discordâncias visa encontrar um particionamento dos vértices em clusters tal que o número de arestas - dentro de clusters mais o número de arestas + entre clusters seja minimizado. A versão de maximização de concordâncias é definida de forma similar. Um survey sobre o problema e suas variações pode ser encontrado em [Becker 2005].

Outro problema de clustering bastante estudado é o problema de Particionamento em Grafos (Graph Partitioning - GP). Nesta variante é dado um grafo $G=(V, E)$ e uma função de pesos $d: E \rightarrow \mathbb{R}$ que representa a distância ou dissimilaridade entre os vértices. Assim como no CC, também se deseja encontrar um particionamento de $V$ em clusters, porém o objetivo é minimizar a soma dos pesos das arestas que estão em um mesmo cluster. Um survey sobre o problema pode ser encontrado em [Buluç et al. 2016].

Neste trabalho, investigamos a variante do CC e GP com pesos nos vértices, e com um custo associado a cada cluster utilizado. Tal cenário surge no contexto de 
logística, em que se deseja realizar o carregamento de uma grande variedade de produtos em contêineres, e se queira minimizar a quantidade de contêineres utilizados, porém considerando que não se deseja carregar produtos muito diferentes juntos, como remédios e veneno, ou eletrônicos e alimentos. Outra aplicação é relacionada com anúncios, em que há um conjunto de propagandas que devem ser dispostas em banners de uma página web ou uma aplicação móvel. Deseja-se encontrar uma disposição que utilize uma quantidade pequena de banners, considerando que propagandas de produtos bem relacionados estejam dispostas no mesmo banner. Nessas situações, é relevante não só a escolha dos elementos que estarão agrupados, como também a quantidade de grupos utilizados.

O restante do texto é organizado da seguinte forma: na Seção 2 descrevemos os problemas que serão abordados formalmente. Na Seção 3 apresentamos formulações matemáticas para tais problemas, e na Seção 4 concluímos com algumas considerações.

\section{Definição dos problemas}

Seja $N=\{1, \ldots, n\}$ um conjunto de $n$ itens, $w: N \rightarrow \mathbb{R}_{+}$uma função que representa o peso de cada item, $d: N \mathrm{x} N \rightarrow \mathbb{R}$ uma função que representa a distância entre pares de itens, com $d_{i i}=0, d_{i j}=d_{j i} \forall i, j \in N$, e $W, c \in \mathbb{R}_{+}$a capacidade e o custo de uso de um cluster, respectivamente. Sem perda de generalidade, consideramos que $w_{i} \leq W$ e $w_{i} \geq w_{j}$ para $i<j$. No problema de GP capacitado (CGP), queremos encontrar uma partição de $N$ dada por $\mathcal{C}=\left\{C_{1}, \ldots, C_{k}\right\}$ tal que $c k+\sum_{C \in \mathcal{C}} \sum_{i, j \in C} d_{i j}$ é mínimo e para todo $C \in \mathcal{C}, \sum_{i \in C} w_{i} \leq W$.

Para o problema de CC capacitado (CCC), consideramos que cada aresta $e \in E$ possui um rótulo + ou - , definido por $s(e)$. Para $S, T \subseteq V$, sendo $E_{+}[S]$ (resp. $\left.E_{-}[S]\right)$ o conjunto das arestas induzidas por $S$ com rótulo + (resp. -), e sendo $\delta_{+}(S, T)=\{(u, v): u \in S, v \in T, s(u, v)=+\}$, o objetivo do CCC é encontrar uma partição de $N$ dada por $\mathcal{C}=\left\{C_{1}, \ldots, C_{k}\right\}$ que minimiza $c k+\sum_{i=1}^{k}\left(\sum_{e \in E_{-}\left[C_{i}\right]} d_{e}\right)+$ $\sum_{i \neq j}\left(\sum_{e \in \delta_{+}\left(C_{i}, C_{j}\right)} d_{e}\right)$, também satisfazendo $\sum_{i \in C} w_{i} \leq W$ para todo $C \in \mathcal{C}$.

\section{Formulações}

Inicialmente, apresentamos uma formulação mais tradicional para o CGP. Para $1 \leq i \leq j \leq n$ e $1 \leq k \leq n$, seja $x_{i j}^{k}$ uma variável de decisão binária que indica se os itens $i$ e $j$ são atribuídos ao cluster $k$ (consequentemente, $x_{i i}^{k}$ indica se o item $i$ pertence ao cluster $k$ ) e $y_{k}$ uma variável que indica se o cluster $k$ está sendo utilizado. Uma formulação para o problema é fornecida a seguir:

$$
\begin{array}{rlr}
\left(F_{T}\right) \min & \sum_{k \in N} \sum_{i \in N} \sum_{j>i} d_{i j} x_{i j}^{k}+c \sum_{k \in N} y_{k} \\
\text { s. a } & x_{i i}^{k} \leq y_{k} & \forall i, k \in N \\
& \sum_{k \in N} x_{i i}^{k}=1 & \forall i \in N \\
& \sum_{i \in N} w_{i} x_{i i}^{k} \leq W & \forall k \in N \\
& x_{i j}^{k} \geq x_{i i}^{k}+x_{j j}^{k}-1 & \forall i, j, k \in N, i<j \\
& x_{i j}^{k} \leq x_{i i}^{k} & \forall i, j, k \in N, i<j \\
& x_{i j}^{k} \leq x_{j j}^{k} & \forall i, j, k \in N, i<j \\
& \text { variáveis } x \text { e } y \text { binárias } &
\end{array}
$$


As restrições (2) impõem que um item é atribuído apenas a clusters utilizados. As restrições (3) garantem que cada item é atribuído a exatamente um cluster. As restrições (4) representam as restrições de capacidade de cada cluster. As restrições (5) indicam que $x_{i j}^{k}=1$ sempre que os itens $i$ e $j$ forem atribuídos ao cluster $k$, enquanto as restrições (6) e (7) fazem com que $x_{i j}^{k}=0$ se o item $i$ ou item $j$ não foram atribuídos ao cluster $k$. Por fim, as restrições (8) se referem às restrições de integralidade das variáveis.

Apresentamos outra formulação para o CGP, baseada nas formulações de arcflow para problemas de empacotamento (veja [Delorme e Iori 2020]). Podemos interpretar a atribuição de itens a um cluster com capacidade $W$ como um empacotamento dos itens em um recipiente (bin) de mesma capacidade. Além disso, a atribuição de itens em um bin pode ser modelada como um problema de caminhos em um digrafo. Seja digrafo $G=(V, A)$, onde $V=\{0, \ldots, W\}$ e $A=A_{i} \cup A_{l}$, com $A_{i}=$ $\left\{(u, v): u, v \in V, \exists j \in N: v-u=w_{j}\right\}$ e $A_{l}=\{(u, u+1): u \in\{1, \ldots, n-1\}\}$. O conjunto de vértices $V$ representa as possíveis posições em que um item pode ser empacotado. Um arco $(u, v) \in A_{i}$ representa um item de tamanho $v-u$ empacotado na posição $u$, e um arco $(u, u+1) \in A_{l}$ representa uma unidade de espaço vazio no bin. Dessa forma, um empacotamento é representado por um caminho de 0 a $W$ em $G$. Com isso, podemos derivar uma formulação pseudopolinomial para o CGP. Considere os itens artificiais 0 e $n+1$, com $w_{0}=w_{n+1}=0$, que são utilizados para representar o início e o fim de um caminho que representa um empacotamento, e seja $N^{\prime}=N \cup\{0, n+1\}$. Seja $x_{i, p, j, p+w_{j}}$ uma variável binária que vale 1 se o item $j$ é empacotado imediatamente após o item $i$ com início na posição $p$ e término na posição $p+w_{j}$, e 0 caso contrário. Note que o quarto índice é redundante e é usado apenas para melhorar o entendimento, não interferindo na quantidade de variáveis. Para reduzir o espaço de possibilidades, os valores $p$ em que um item pode ser atribuído é limitado pelos padrões normais, definido como $\mathcal{N}=\left\{x: x \leq W, x=\sum_{i \in N} w_{i} \epsilon_{i}, \epsilon_{i} \in\{0,1\} \forall i \in N\right\}$, e representa o conjunto de possíveis coordenadas em que um item pode ser atribuído se todos os itens forem deslocados o máximo possível para a esquerda. Seja também $y_{i j}$ uma variável binária que indica se o item $j$ é empacotado imediatamente após o item $i$. Tal variável é redundante com as variáveis $x$, como será visto na formulação a seguir, e é utilizada apenas para simplificar o modelo. Por fim, sejam as variáveis binárias $z_{i j}$, que indica se os itens $i$ e $j$ estão no mesmo $\operatorname{bin}$, e $b_{i}^{k}$, que indica se o item $i$ foi atribuído ao bin $k$. Consideramos apenas as variáveis $x, y$ e $z$ com $i<j$. A formulação, denotada por $F_{\mathrm{CGP}}$, é dada em (9) - (19).

A função objetivo (9) minimiza a soma dos pesos das arestas que estão em um mesmo cluster, mais o custo de uso dos clusters, pois como todo empacotamento é feito a partir da posição 0 , cada caminho que parte do vértice 0 equivale a um cluster na solução. As restrições (10) relacionam as variáveis $y$ com as variáveis $x$. As restrições (11) e (12) impõem que todo item é precedido e sucedido por exatamente um outro item (que pode ser um dos itens 0 ou $n+1$ ), respectivamente. As restrições (13) se referem às restrições de conservação de fluxo. As restrições (14) garantem que todo item pertença a exatamente um bin. As restrições (15) fazem com que o índice do primeiro item que é alocado em um bin seja usado como índice do bin ao qual ele pertence, a fim de evitar simetrias. As restrições (16) indicam que se o item $i$ precede $j$ em algum bin, então $i$ e $j$ estão no mesmo bin, e as restrições (17) triangulam as variáveis $z$. As restrições (18) fazem com que $b_{j}^{k}$ seja 1 se os itens $i$ e $j$ estão no mesmo bin e o item $i$ está no bin $k$. Além disso, esse conjunto de restrições é responsável por fixar $z_{i j}$ em 0 se os itens $i$ e $j$ estão em bins 
diferentes. Tal fixação é importante quando $d_{i j}<0$. Se todas as arestas possuem peso não-negativo, podemos remover as variáveis $b$ e as restrições (14), (15) e (18). Por fim, as restrições (19) se referem às restrições de integralidade das variáveis.

$$
\begin{aligned}
& \left(F_{\mathrm{CGP}}\right) \\
& \min \sum_{i \in N} \sum_{j>i} d_{i j} z_{i j}+c \sum_{i \in N} x_{0,0, i, w_{i}} \\
& \text { s. a } \quad y_{i j}=\sum_{\substack{p \in \mathcal{N} \\
w_{i} \leq p \leq W-w_{j}}} x_{i, p, j, p+w_{j}} \\
& \sum_{i<j} y_{i j}=1 \\
& \sum_{j>i} y_{i j}=1 \\
& \sum_{\substack{i<j \\
p-w_{j} \geq w_{i}}} x_{i, p-w_{j}, j, p}-\sum_{\substack{k>j \\
p+w_{k} \leq W}} x_{j, p, k, p+w_{k}}=0 \\
& \sum_{k \in N} b_{i}^{k}=1 \\
& b_{i}^{i}=y_{0 i} \\
& z_{i j} \geq y_{i j} \\
& z_{i k} \geq z_{i j}+z_{j k}-1 \\
& b_{j}^{k} \geq b_{i}^{k}+z_{i j}-1 \\
& \forall i, j \in N^{\prime}, i<j \\
& \forall j \in N \\
& \forall i \in N \\
& \forall j \in N, p \in \mathcal{N} \\
& \forall i \in N \\
& \forall i \in N \\
& \forall i, j \in N, i<j \\
& \forall i, j, k \in N, i<j<k \\
& \forall i, j, k \in N, i<j
\end{aligned}
$$
extremos estão dentro de um mesmo cluster. Dessa forma, torna-se simples adaptar $F_{\mathrm{CGP}}$ para resolver o CCC, bastando alterar a função objetivo da forma apresentada em $F_{\mathrm{CCC}}$.

$$
\begin{aligned}
\left(F_{\mathrm{CCC}}\right) \min & \sum_{(i, j) \in E_{-}} d_{i j} z_{i j}+\sum_{(i, j) \in E_{+}} d_{i j}\left(1-z_{i j}\right)+c \sum_{i \in N} x_{0,0, i, w_{i}} \\
\text { s. a } & (10)-(19)
\end{aligned}
$$

\section{Considerações finais}

Apresentamos duas formulações para o CGP, $F_{T}$ e $F_{\mathrm{CGP}}$. Experimentos computacionais indicam que a formulação $F_{\mathrm{CGP}}$ geralmente encontra soluções ótimas mais rapidamente do que $F_{T}$ em cenários com grafos esparsos e com custos de cluster altos comparados com os valores de dissimilaridade entre os itens. Outra característica relevante em relação à $F_{\mathrm{CGP}}$ é a facilidade de adaptação da formulação para outros problemas de clustering, como mostrado em $F_{\mathrm{CCC}}$. Ademais, ela pode ser adaptada para situações em que há necessidade de se colocar um separador entre itens adjacentes, isto é, se os itens $i$ e $j$ são empacotados lado a lado, então um separador de tamanho $c_{i j}$ deve ser colocado entre eles. Para tal, podemos adaptar as variáveis $x$ de $x_{i, p, j, p+w_{j}}$ para $x_{i, p, j, p+w_{j}+c_{i j}}$.

Agradecimentos. $\mathrm{O}$ presente trabalho foi realizado com apoio do CNPq (Proc. 425340/2016-3), da FAPESP (Proc. 2015/11937-9, 2016/01860-1 e 2019/12728-5) e do Quinto Andar.

\section{Referências}

Becker, H. (2005). A survey of correlation clustering. Advanced Topics in Computational Learning Theory, pages 1-10.

Buluç, A., Meyerhenke, H., Safro, I., Sanders, P., e Schulz, C. (2016). Recent Advances in Graph Partitioning, pages 117-158. Springer International Publishing, Cham.

Delorme, M. e Iori, M. (2020). Enhanced pseudo-polynomial formulations for bin packing and cutting stock problems. INFORMS Journal on Computing, 32(1):101-119. 